\title{
Directed Growth of Biomimetic Microcompartments
}

\section{Ivan Ivanov ${ }^{1 *}$, Rafael B. Lira ${ }^{2}$, T-Y. Dora Tang ${ }^{3}$, Titus Franzmann ${ }^{3}$, Adam Klosin ${ }^{3}$, Lucas}

2 Caire da Silva ${ }^{4}$, Anthony Hyman ${ }^{3}$, Katharina Landfester ${ }^{4}$, Reinhard Lipowsky ${ }^{2}$, Kai

\section{Sundmacher ${ }^{1}$, Rumiana Dimova ${ }^{2 *}$}

$4{ }^{1}$ Max Planck Institute for Dynamics of Complex Technical Systems, Process Systems Engineering,

5 Sandtorstrasse 1, 39106 Magdeburg, Germany

$6{ }^{2}$ Max Planck Institute of Colloids and Interfaces, Theory and Bio-Systems, Science Park Golm,

714424 Potsdam, Germany

$8 \quad{ }^{3}$ Max Planck Institute of Molecular Cell Biology and Genetics, Organization of Cytoplasm \&

9 Dynamic Protocellular Systems, Pfotenhauerstrasse 108, 01307 Dresden, Germany

$10{ }^{4}$ Max Planck Institute for Polymer Research, Physical Chemistry of Polymers, Ackermannweg 10, 1155128 Mainz, Germany

\begin{abstract}
Contemporary biological cells are sophisticated and highly compartmentalized. Compartmentalization is an essential principle of prebiotic life as well and a key feature in bottom-up synthetic biology research. In this review, we discuss the dynamic growth of compartments as an
\end{abstract}




\section{Directed Growth of Biomimetic Microcompartments}

essential prerequisite for enabling self-reproduction as a fundamental life process. We focus on micron-sized compartments due to their cellular dimensions. Two types of compartments are considered, membraneless droplets and membrane-bound microcompartments. We review growth mechanisms of aqueous droplets such as protein (condensates) or macromolecule-rich droplets (aqueous two phase systems) and coacervates, for which growth occurs via Ostwald ripening or coalescence. For membrane-bound compartments, we consider vesicles, composed of fatty acids, lipids or polymers, where directed growth can occur via fusion or uptake of material from the surrounding. The development of novel approaches for growth of biomimetic microcompartments can eventually be utilized to construct new synthetic cells.

\section{Introduction}

Despite the long and ongoing debate about the essence of life, there is a widespread consensus about some common features of all living systems. While some phenomena, such as metabolism, remain arguable benchmarks (and place viruses in the twilight zone), it is widely recognized that the formation of micro- and nanocompartments is an essential ingredient of all forms of life. This spatial constraint serves numerous purposes, including the segregation and protection from the environment (to allow for individuality and maintenance), the establishment of gradients (to enable and make use of out-of-equilibrium conditions), and the role of two- and three-dimensional confinement for selforganization phenomena, all of which serve to overcome the overall "dilution problem". In order to fulfil another cornerstone of life - proliferation - compartments also need to change with time by fusion, growth, and division. In particular, the dynamic growth of compartments is an essential prerequisite for enabling self-reproduction as a fundamental life process, both in simplistic systems such as droplets or fatty-acid based vesicles, as well as for lipid vesicle compartments with membranes that resemble the biomembranes of today's cells. In this context, we argue that growth deserves more attention, not only because growth precedes division but also because of the difficulty to realize growth compared to division, especially in the case of lipid vesicles, where budding and 


\section{Directed Growth of Biomimetic Microcompartments}

\footnotetext{
${ }^{1}$ We conceptually include examples of fatty acid vesicles as presumable predecessors, as well as vesicles made of synthetic chemicals as prospective successors of today's cells.
} 


\section{Directed Growth of Biomimetic Microcompartments}

672 Coacervates and protein-rich droplets - volume and area grow simultaneously

In the 1920's Oparin hypothesized that membrane-free liquid-like droplets formed via complex coacervation could have been precursors to modern cells $[5,6]$. Extensive research has demonstrated that small molecules and oppositely charged polymers can phase separate to form structures with emerging liquid-like properties. More recently, it was discovered that certain types of proteins undergo intracellular phase separation to form membrane-free/membraneless liquid-like protein-rich droplets. Both of these membraneless microcompartments represent interesting systems to circumvent the coupling between volume and surface area, and their austerity in comparison to membrane-bound microcompartments has qualified them as useful models to demonstrate compartment formation and growth. The relevance of membraneless compartments to bottom-up synthetic biology, regardless of their chemical composition, remains undisputed, since they successfully represent or mimic certain biological aspects. Thus, phase-separated droplets represent useful models of self-organized entities, independent of the natural or man-made origin of the macromolecules. Form a protobiology perspective, coacervates made of synthetic polymers are logically excluded as potential ancestors of today's cells, but such coacervates have played an important role in the history of protocells, starting with the first experiments on gum arabic and gelatin.

\subsection{Protein-rich droplets}

Compartmentalization is a hallmark of the eukaryotic cell. It enables cells to spatially separate their complex biochemistry into microreactors. Well-known examples of compartments are the mitochondrion and the nucleus, both of which are surrounded by lipid bilayers. However, in recent years it has become clear that the eukaryotic cytoplasm is further organized by compartments that lack membranes. These compartments have been termed biomolecular condensates, also known as 


\section{Directed Growth of Biomimetic \\ Microcompartments}

membraneless organelles [7]. Intracellular condensates form by the process of liquid-liquid phase

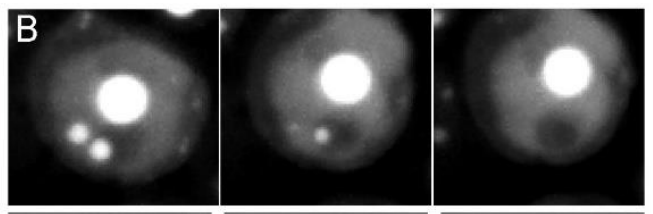

separation [8-13]. Phase separation is a highly cooperative and concentration-dependent process: above a certain saturation concentration, a solution becomes unstable and demixes to form two or more distinct co-existing aqueous phases, a protein-dense and a protein-poor phase that continuously exchange molecules and maintain a steep concentration gradient across their interfaces. Recent work suggests, that the formation of biomolecular condensates is primarily driven by RNA and proteins that exhibit a large degree of intrinsic disorder and/or multivalency. Among the growing list of proteins that undergo liquid-like phase separation are the well-studied P-granule proteins LAF1 and PGL-3 [14, 15], the stress granule proteins FUS and hnRNPA1 [16-18], the centrosome protein SPD5 [19], as well as the nucleolar proteins Fib1 and Npm1 that form immiscible phases to organize the nucleolus [20]. When expressed in a test tube, these proteins readily phase-separate to form twophase systems at physiological concentrations. These protein-rich droplets grow by taking up material from the environment (Ostwald ripening) or via coalescence of two smaller droplets to form a larger one; see Figure 1. The condensates exhibit liquid-like characteristics, such that they can coalesce, wet surfaces and deform under shear stress. The dynamics of these processes is governed by internal viscosity and surface tension $[8,14,21]$. Importantly, condensates display selectivity, and client molecules such as interacting proteins or RNA will partition into the condensates.
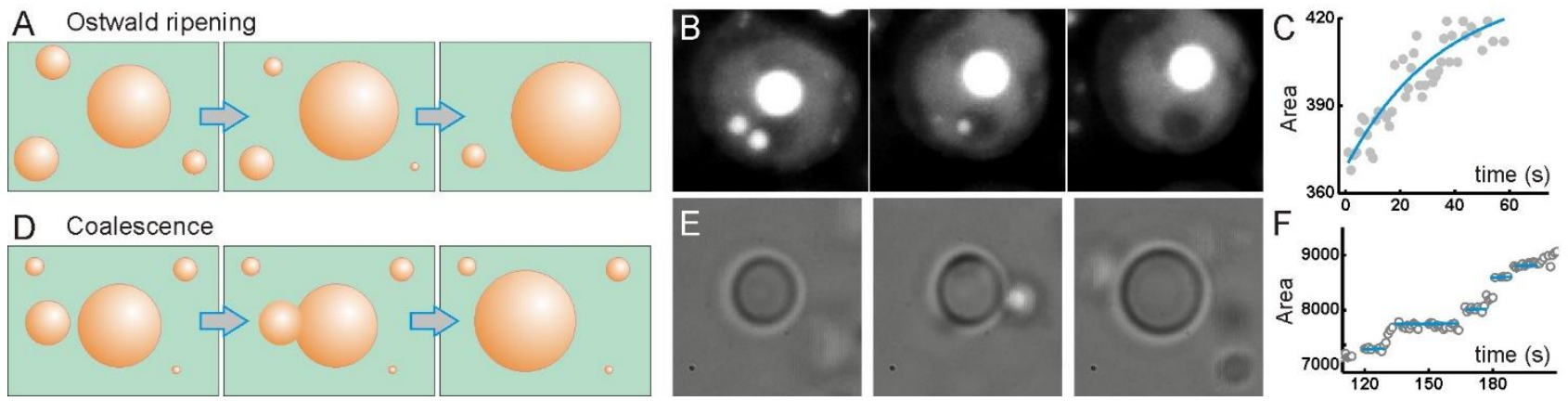

Figure 1. (A) Protein-rich droplets can grow by the uptake of material from the surrounding and at the expense of smaller droplets (Ostwald ripening). (B) Still fluorescence images from a time-lapse 
movie of a Hela cell expressing the YFP-labelled disordered region of the RNA helicase DDX4 (unpublished data). The disordered region forms an intracellular liquid-like droplet. With time, the bigger droplet grows, whereas the smaller droplets shrink and eventually disappear. (C) Quantification of the bigger droplet area shown in (B) as a function of time (unpublished data). (D) Growth of liquid-like protein droplets can also occur via coalescence upon encounter of two or more droplets that merge to form a bigger one. (E) Still bright field images from a time-lapse movie of protein droplets formed from the prion protein Sup35 [22]. The protein droplet was held with an optical tweezers and brought in the vicinity of other droplets, which coalesce leading to growth with time. (F) Quantification of the droplet area shown in (E) as a function of time. The step-wise increase in area is indicative of coalescence.

Recent work has identified that the saturation concentration of the FET family of proteins (FUSEWSR1-TAF15 protein family) is quantitatively determined by the associative cation-pi interactions

between segregated tyrosine and arginine sticker residues [23]. Other types of interactions, such as pi-pi and electrostatics between charged residues, play important roles as well [24]. For instance, the disordered region of the condensate protein helicase DDX4 displays a great degree of segregated charges [25]. Importantly, weak multivalent interactions underlie the nucleation process [11].

In the context of the cell, these systems are driven away from equilibrium, e.g. by chemical reactions. However, in the test tube, droplet growth continues until the thermodynamic equilibrium has been reached. Accordingly, mechanisms must exist that regulate the nucleation as well as the size and the mechanical properties. Liquid-like condensates can also undergo liquid-to-solid transitions [18, 22, 26, 27]. For FET family proteins, the composition of interspacing residues (so-called spacers) that segregates the stickers plays an important role in modulating the interaction strength and thus the liquid-to-solid transition [23]. The liquid-to-solid transition of the centrosome forming protein SPD-5 may be mediated by coiled-coil domains to establish a force-resistant meshwork for microtubulemediated chromosome segregation [19]. Coupling phase separation to gelation may provide an ideal size-determining mechanism. Moreover, coupling the phase behavior to biochemical reactions that drive the system away from equilibrium establishes control over nucleation, droplet growth and under certain circumstances may even provide a mechanism for droplet division [28, 29]. Phase separation is exquisitely sensitive to environmental changes, including temperature, ionic strength or 


\section{Directed Growth of Biomimetic Microcompartments}

$\mathrm{pH}$, parameters that also fluctuate within cells [30,31]. Cells have harnessed the phenomenon of

phase separation to orchestrate complex processes and evolved precise mechanisms to control nucleation of biomolecular condensates. Taken together, it is now possible to predict the saturation concentration, as well as the material constants for minimal proteinaceous systems. Linking synthetic phase separation units with enzyme functions provides an opportunity to organize and polarize biochemical reactions within a synthetic cell.

\subsection{Polymer-rich droplets}

Coacervate microdroplets are generated from associative liquid-liquid phase separation (coacervation) processes between oppositely charged polymers or small highly charged molecules. They form from synthetic polymers (PDDA, polyethylemine, etc.) [32, 33], as well as from biologically relevant molecules including proteins (BSA) [34], polypeptides (polylysine, polyarginine) $[35,36]$, nucleic acids (DNA, RNA) $[33,37,38]$, and co-factors (ATP, FAD, NAD) [39]. In comparison, dissociative liquid-liquid phase separation into two aqueous phases can be triggered in solutions of neutral macromolecules such as polyethylene glycol (PEG) and dextran, and more recently, this process was also established in the closed compartment of lipid vesicles [40, 41]. It is commonly considered that the general mechanism of phase separation occurs via two steps: an enthalpic contribution to the free energy from the electrostatic interaction between the molecules (in the case of charged polymers), which draws the molecules towards each other, and an entropic driving force from the rearrangement of ions and water leading to a lowering of the Gibbs free energy and the formation of membraneless, chemically enriched microdroplets. It has been proposed that this mechanism is analogous to liquid-liquid phase separation in biology $[42,43]$. The surface tension of coacervates is low, between $1 \mu \mathrm{N} / \mathrm{m}-1 \mathrm{mN} / \mathrm{m}$ [44], and even lower for aqueous two-phase systems of neutral polymers [45]. The droplets will grow in size over time via coalescence events [46]. The 


\section{Directed Growth of Biomimetic \\ Microcompartments}

rate of coalescence and therefore growth can be tuned by the overall charge ratios of the coacervate

${ }_{6}^{163}$ components.

Studies have shown that polymer-rich microdroplets will selectively partition a range of different molecules leading to molecular localization and further chemical enrichment [39, 47-49]. Moreover, enzymes, which partition into the microdroplets maintain activity within the highly charged and crowded interior [50, 51]. Enzymes capable of phosphorylation and dephosphorylation have led to cycles of growth and degradation of the coacervate droplets [37, 52]. In addition, growth and decay of coacervate droplets can be instigated by switching the $\mathrm{pH}$ of the solution by bubbling carbon dioxide and ammonia through the dispersion of coacervate droplets [39]. These results indicate that the physical parameters such as molar ratio of polymers or $\mathrm{pH}$ and temperature of coacervate droplets can be used to tune and drive droplet growth and disassembly. Additionally, the ability of membraneless coacervate droplets to partition and support enzyme reactions could suggest that these systems could be plausible models for predicted growth and division cycles driven by flux of molecules across the interface [29].

\section{Fatty acid vesicles as primitive protocells - membrane formation due to fast equilibrium}

The vesicular (membrane) systems represent another compartment type with the same structural features as living cells. Worth noting with respect to the field of minimal cells, and growth in particular, are the pioneering works of Luisi and Szostak [53, 54], which mainly relied on fatty acid micelles and vesicles. The latter have been investigated extensively as protocell models because fatty acids have been proposed to be prebiotically relevant due to their structural simplicity compared to phospholipids [55]. Therefore, the spontaneous uptake of fatty acids into preformed vesicles has often been considered as a primitive growth mechanism. As far as structural simplicity is concerned, the emphasis on fatty acids is analogous to the RNA hypothesis (single-/double-stranded vs. one or 


\section{Directed Growth of Biomimetic \\ Microcompartments}

two hydrophobic tails) but the quest for the origin of life is intrinsically speculative and we will refrain from statements about the evolutionary relevance of these compartments. Regardless of this open question, fatty acid vesicles and fatty acids have served as rewarding models for mimicking life processes, including growth.

We also note that the majority of studies with fatty acids employed small $(\sim 30-50 \mathrm{~nm})$ and large $(\sim 100-200 \mathrm{~nm})$ unilamellar vesicles - below the limit of optical resolution - which poses the question whether those are relevant protocell models from another perspective, namely their small size. A workshop dedicated to defining the minimal compartment size of life, related to possibilities for extraterrestrial life and nanobacteria, dealt with this question 20 years ago, see proceedings [56]. The biologists view regarding the threshold size of primitive compartments was set to diameters of $250 \pm 50 \mathrm{~nm}$ determined by a requirement for the compartment to accommodate minimum amount of necessary proteins, genes and ribosomes. Others views were more speculative suggesting $\sim 50 \mathrm{~nm}$ as the minimal size required to sustain potential forms of primordial life. The latter notion was supported by reports on enhanced protein expression in $100 \mathrm{~nm}$ liposomes [57]. Considered from a simplistic chemoton perspective, smaller size implies higher surface-to-volume ratio, which is associated with enhanced inward flux (proportional to the surface) of membrane precursors and other metabolites and thus could be considered as beneficial. However, other factors such as packing, curvature, etc., could result in micrometer size (as in modern cells) optimal for a specific functions. In this review, we focus on biomimetic microcompartments, while we do not aim to set the size limits of protocells. However, we include reports on nanocompartments as well, due to their historical and mechanistic significance. Furthermore, microcompartments such as giant unilamellar vesicles (GUVs) [58-61] have proven to be a practical system, whose properties, growth and response to external factors can be monitored and manipulated directly under a microscope. 
Compared to phospholipids, fatty acids exhibit a very quick exchange between the membrane leaflets

as well as faster exchange kinetics between the vesicle and the solution. In addition, stabilizing head group interactions are more important, which endows distinct properties, such as growth and selfreproduction of vesicles [62-64]. Due to their single-chain structure, the concentration of monomers in equilibrium with vesicles is significantly higher than for phospholipids, which enables fast flipflop and exchange of molecules; Figure 2A. These beneficial properties have qualified fatty acids as a prominent system for studying protocells, including the demonstration of membrane growth [62]. A notable phenomenon, observed during the growth of oleate vesicles, was the so-called matrix effect, which exemplified itself when a seed of preformed vesicles was added to the micellar oleate solution, resulting in a narrow size distribution, corresponding to the size of the seed [65]. The growth of oleate vesicles was further investigated in detail, which lead to kinetic [66] and molecular dynamics [67] models. The growth process was also demonstrated under flow [68], whereby filamentous microcompartments were formed, and also employed as a mechanism for ribozyme activation [69] to manifest a form of homeostasis. In addition, the filamentous growth of multilamellar oleate vesicles $(\sim 4 \mu \mathrm{m})$ was used to mimic the full proliferation cycle of growth and division [70] and the chemistry was extended to other surface-active molecules beyond oleate [71].

While fatty acids and surfactants are efficiently incorporated into existing membranes due to their optimal physicochemical properties, phospholipids - the membrane constituents of modern living cells - form structures, which are stable over a wider range of concentrations (phospholipids exhibit lower critical micelle concentration, $\mathrm{CMC}$, compared to fatty acids). With respect to this distinction, the addition of oleate to preformed phospholipid vesicles was used in other attempts to mimic selfreproduction as an intermediate approach. Growth and division has been shown and investigated in detail in several examples of fatty acid incorporation into smaller liposomes [72-74] and the method has been later on scaled up to GUVs $[75,76]$. In a similar fashion, the addition of detergents and 


\section{Directed Growth of Biomimetic Microcompartments}

${ }_{4}^{3} 32$ other water-soluble membrane active molecules at low concentration leads to their intercalation into

8234 depends on whether the inserting molecules exhibit fast or slow flip-flop. Fast flip-flop induces a 9

large, visible increase in membrane area and fluctuations $[75,77,78]$. Slow flip-flop molecules induce an increase in the membrane spontaneous curvature due to their asymmetric incorporation into the outer leaflet of the membrane, resulting in tubulation [79-81], increased membrane tension and eventually rupture $[75,77]$. Interestingly, the incorporation of simpler amphiphilic molecules or micelles leads to vesicle growth, often followed by division $[70,82]$, which is in line with predictions for phospholipids [83]. The limitation of detergent-like molecules is that at high concentrations, they lead to membrane permeabilization and eventual solubilization, depending on the membrane phase state and the CMC of the surfactant $[84,85]$. In general, although this mixed approach provides a tangible system for studies of liposome growth, there is major conceptual drawback in the context of minimal cells - such microcompartments would lose their chemical identity over several generations and therefore could not sustain a continuous life cycle. The same drawback applies to another approach, in which blends of phospholipids and fatty acids or cationic surfactants were fused by attractive electrostatic interactions [86]. 

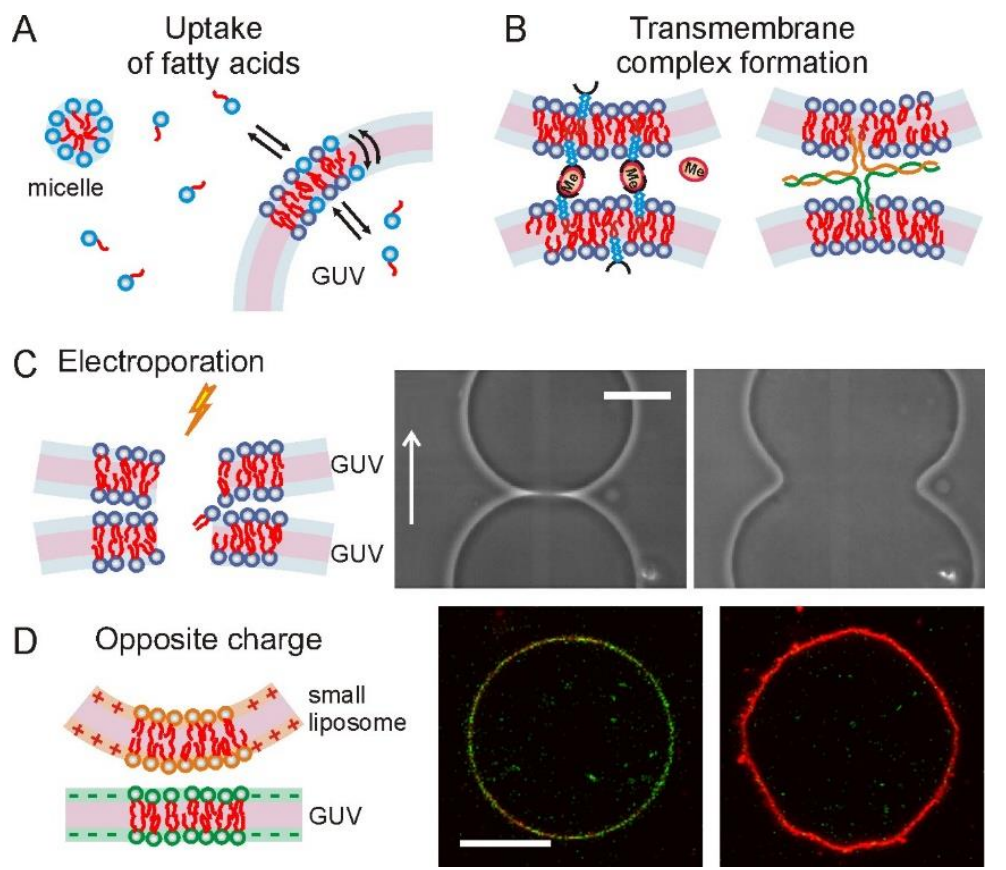

Figure 2. Some approaches for establishing growth of giant vesicles. (A) Growth via uptake of fatty acid molecules, which remain in dynamic exchange with the external and internal GUV solution and exhibit fast flip-flop across the membrane. (B) Growth via fusion mediated by the formation of transmembrane metal-ligand complex (left) or fusion protein mimetics (right). (C) Electrofusion relies on poration of two opposing membranes exposed to strong electric fields. The phase contrast snapshots on the right illustrate the fusion of two GUVs brought in contact and aligned by means of AC field, followed by the application of a DC pulse; reproduced from [87]. Copyright (2006) National Academy of Sciences. The white arrow indicates the direction of the field. (D) Growth of giant vesicles can be established by efficient fusion of small liposomes with the GUV, where the two membrane types are oppositely charged. Fusion is illustrated with the cartoon (left) and the confocal images (right) of one negatively charged GUV (green) exposed to a solution of positively charged small liposomes (red). Upon fusion, Förster resonance energy transfer leads to decrease in the red signal and the GUV area increases significantly [88]. The change occurs within seconds. Reprinted from [88] Copyright (2019), with permission from Elsevier. Scale bars in (C, D) represent $20 \mu \mathrm{m}$.

\section{Growth of lipid vesicles - a difficult task, solved by fusion}

The system of highest interest for mimicking the proliferation of living cells is the phospholipid vesicle, which grows as a result of the incorporation of phospholipids in its membrane and eventually divides. The importance of liposomes arises from the chemical analogy with modern cell membranes. The cellular self-reproduction found in nature involves very complex biochemical interactions and 


\section{Directed Growth of Biomimetic Microcompartments}

machineries. According to the reductionist concept, research in the context of protobiology and 5 bottom-up synthetic biology aims to establish this phenomenon in a minimal system, based on

simpler physicochemical mechanisms in a proof-of-concept for the origin and understanding of life.

So far, however, growth of pure lipid vesicles has proved to be a challenge. Due to the difficulty of experimental realization of the membrane formation subsystem, however, theoretical studies have preceded practical examples in the case of phospholipids. Different kinetic [89] and thermodynamic [83, 90, 91] aspects have been considered and summarized together with other, chemoton-like approaches in a recent review [92]. On the practical side, the self-reproduction of a biomembrane, i.e. a phospholipid vesicle building its own membrane, has been addressed several times, but with only modest success so far.

\subsection{Growth via uptake of synthesized membrane components}

Efforts for synthesizing membrane components in liposomes date back to the early 90s. In particular, Luisi and coworkers assembled a four-enzyme cascade for phosphatidylcholine (PC) synthesis and deduced its incorporation into the membrane based on geometrical considerations (eventually the liposome size decreased, which was ascribed to the higher spontaneous curvature triggered by the newly synthesized short-chain PC - a partially undesired outcome with respect to growth) [93]. The authors did not unequivocally determine, whether the enzymes were present in the interior, whereby significant dimensional limitations could arise from the small liposome radius $(23-26 \mathrm{~nm})$, but they ascertained the enzyme association with the membrane to maintain an active form. In another study, the synthesis of phosphatidic acid by acyltransferases, expressed via cell-free methods in vesicles, was demonstrated but no growth was observed [94]. The latter observation was ascribed to the low amount of product due to the limited encapsulation, which will be difficult to overcome unless efficient transport mechanisms for the various precursors are established. Recently, this cell-free approach was extended to a more comprehensive pathway, starting from acyl-CoA and glycerol-3- 


\section{Directed Growth of Biomimetic \\ Microcompartments}

phosphate, and the liposome compartments were equipped with $\alpha$-hemolysin to facilitate uptake of

small polar molecules [95]. Although the authors did not specifically follow the vesicle growth, they identified practical barriers causing the low lipid synthesis rate, speculated about transport mechanisms for acyl-CoA and discussed relevant crowding and confinement effects. Another example in a similar direction was the biochemical synthesis of palmitate based on a FAS type I enzyme, encapsulated in liposomes [96], where vesicle growth has indeed been observed but the incorporation of foreign membrane components remains a conceptual problem.

To circumvent protein synthesis issues, inherent to cell-free systems, an eight-enzyme cascade for the biosynthesis of phospholipids from fatty acids and glycerol 3-phosphate as building blocks has recently been assembled in vitro [97]. In the latter study, the enzymes and precursors were not encapsulated and synthesis occurred outside the liposomes. Experiments measuring the dequenching of rhodamine dye showed an approximately $30 \%$ membrane expansion, which was subsequently limited by the depletion of substrate. The growth was ascribed to the incorporation of oleic acid into the preformed liposomes, while the subsequent biosynthetic conversion to phosphatidic acid did not contribute to further expansion. Despite these limitations, the reconstitution of the biochemical membrane formation machinery is undisputedly a landmark in the area of bottom-up synthetic biology.

The continuous search for more realistic growing protocell models has led to the use of phospholipidlike molecules, supplied from outside, instead of internally synthesized. The Sugawara group demonstrated growth, division and amplified DNA distribution in daughter vesicles [98]. This was achieved by adding a cationic precursor, which was hydrolyzed into the membrane lipid by an embedded catalyst. Notably, DNA amplification enhanced the efficiency of both growth and division. However, the elegant process was ultimately exhausted because it was limited by the consumption of DNA precursors inside the vesicles and the dilution of membrane catalyst and 


\section{Directed Growth of Biomimetic \\ Microcompartments}

${ }_{4}^{3} 18$ phospholipids. Similar approaches were undertaken by the replacement of the complex biochemical

lipid synthesis pathway with a self-reproducing autocatalyst, which resulted in triazole phospholipids

formation and membrane growth [99]. Both cases seem to function with aggregates and multilamellar vesicles, and the exotic chemistry of the membranes might hinder interactions with some biological species (e.g. sensitive membrane proteins), thus potentially limiting other applications in the context of minimal cells.

\subsection{Growth via vesicle fusion}

In the case of authentic phospholipids, the only established and practical approach for achieving growth until now seems to be via vesicle fusion. Membrane fusion is a ubiquitous process in biology and it is fundamental for a number of cellular processes. It involves merging of two otherwise separated membranes, forming a compartment, whose area is the sum of the two fusing bilayers. In other words, increase in membrane area inevitably accompanies fusion, although the former is scarcely reported because the vast majority of fusion assays are insensitive to it. Driving forces of various nature have been employed to trigger fusion, some approaches are illustrated in Figure 2. These range from simple physicochemical triggers such as electrostatic interactions [100-102] and volume depletion [103] to biochemical approaches, relying on natural protein fusion machinery [104], as well as combinations thereof [105]. Furthermore, fusion methods have been expanded to biomimetic strategies $[87,106]$ in addition to more exotic approaches, involving the use of light (to heat nanoparticles [107] or isomerize azocompounds [108]) or electric fields [87, 109, 110] to perturb and porate the bilayer. In some of the examples fusion has led to spontaneous budding, correlating to predictions made by theoretical models [90], which has been put in the context of growth and division cycles for self-reproduction [109]. Presumably, the simplest mechanism to induce membrane fusion is based on membrane tension as has been elucidated by molecular simulations [111]. 
3341 Membrane fusion of small liposomes has been used for decades [112, 113] and only more recently

fusion using giant vesicles has been reported [114]. In cells, membrane fusion is mediated by specific interactions between fusion proteins and such fusion reactions have been reconstituted in synthetic systems, in which small liposomes fuse with giant vesicles $[104,115,116]$. However, possibly due to the low fusion efficiency of protein-reconstituted systems or the low protein density, vesicle growth has not been detected. Fusion mediated by $\mathrm{pH}$-sensitive lipids has also resulted in undetectable area increase [117]. In contrast, when mediated by high charge density, the fusion of positively-charged liposomes induces extensive area increase of the negative GUVs [88, 118], see also Figure 2D. Although very efficient, the process is limited by eventual charge neutralization.

The fusion of two GUVs can be induced and observed also in a more controllable way. This can be achieved by bringing a pair of vesicles in contact by manipulation with ultramicroelectrodes [119], by trapping them within microfluidic devices [120], by applying an electric field [87, 110], or by direct manipulation using micropipettes [87, 121] or optical tweezers [107, 122]. After initial contact, fusion can be triggered by the application of a strong DC pulse [87, 123], via ligand-mediated ion interactions [87], transmembrane domains of fusogenic proteins [124] or through localized nanoparticle-mediated [107] or laser-mediated [122] heating. The common feature of all these studies is that an increase in membrane area is clearly observable. Although GUV fusion may result in visible area increase, the resulting area has never been quantified and fusion efficiency has been determined by changes in the measured fluorescence rather than the measured volume changes of the vesicle to characterize vesicular growth. Generally, the excess area results in increased membrane fluctuations and formation of membrane folds as a consequence of volume expansion lagging behind the area growth. The GUV-GUV fusion approach in its variations has been used to mix chemical precursors for nanoparticle formation inside the vesicular compartment $[119,122,123]$, to 
controllably obtain vesicles with well-defined compositions [121], and to unravel the formation of

the hemifusion diaphragm $[124,125]$ and the rapid nature of the fusion neck expansion [87].

Importantly, fusion is not limited to pure phospholipid GUVs, but can be also extended to fusion of GUVs and cells $[126,127]$ or GUVs made of synthetic polymers. In contrast to liposomes, fusion of polymersomes is limited by the restricted mobility and high stability of the polymer membranes [128-130] associated with slowed down polymer rearrangement, which is a prerequisite for processes such as fusion. Some strategies have been successful in inducing fusion events in polymer GUVs, including exposure of vesicles to osmotic pressure gradients [128], ultrasound treatment [131], membrane perturbation by azobenzene photoisomerization [132] and addition of membrane disruptive agents into the dispersing medium [133].

In relation to bottom-up synthetic biology and protobiology, although fusion has been traditionally considered from a biophysical and biochemical perspective, spontaneous liposome growth could in principle occur (or could have occurred in the evolutionary history) by the discrete steps of vesicle fusion [134]. In addition, apart from a mechanism for increasing the membrane area, it has been speculated as a scenario, in which two systems combine their properties and thus increase the degree of biocomplexity [135], for instance considering that vesicular trafficking is a major transport mechanism for proteins in natural living cells [136]. This aspect was used to supply feeding components and trigger gene expression in the case of fusion of oppositely charged vesicles [137].

\section{Synthetic chemistry enables new potential approaches for growth}

In parallel to the continuing efforts to form and grow new types of membraneless microcompartments, enable efficient fusion via new approaches, and ideally reconstitute the natural machinery for phospholipid synthesis, there is another possible pathway, which brings synthetic chemistry in the foreground of bottom-up synthetic biology. This aspect has been already partially 
addressed by the demonstration of life-like properties of dichloromethane and nitrobenzene oil

droplets, which grow, divide and partition cargo $[138,139]$. The versatility of synthetic reactions was

also used to augment phospholipid vesicles and make them grow [98, 99] as noted above. With respect to this, advances in the preparation of functional amphiphilic block copolymers provide powerful synthetic tools for the engineering of artificial compartments that could grow upon stimulation by light, temperature or $\mathrm{pH}$. Here the stimuli would induce the build-up of membrane stresses that could relax through fusion events. In this way the chemistry of living systems could be extended far beyond natural building blocks, enabling not only the reverse engineering or reengineering, but also the de novo engineering of life. Besides a powerful toolbox, which may enable futuristic applications, the latter concept of synthetic (in its conventional semantics of chemical) has resorting implications on the understanding of life as a display of self-organization and will expand our search criteria for other forms of life.

\section{Conflict of Interest}

The authors declare that the research was conducted in the absence of any commercial or financial relationships that could be construed as a potential conflict of interest.

\section{$7 \quad$ Author Contributions}

All authors listed have made a substantial, direct and intellectual contribution to the work, and approved it for publication.

\section{$8 \quad$ Funding}

This work is part of the MaxSynBio consortium, which is jointly funded by the Federal Ministry of Education and Research of Germany (BMBF) and the Max Planck Society (MPG).

\section{$9 \quad$ References}

[1] T. Ganti, Organization of Chemical-Reactions into Dividing and Metabolizing Units Chemotons, Biosystems 1975, 7, 15-21.

[2] T. Ganti, Biogenesis itself, Journal of Theoretical Biology 1997, 187, 583-593.

[3] P.L. Luisi, Autopoiesis: a review and a reappraisal, Naturwissenschaften 2003, 90, 49-59.

[4] F.G. Varela, H.R. Maturana, R. Uribe, Autopoiesis: the organization of living systems, its characterization and a model, Biosystems 1974, 5, 187-196.

[5] A.I. Oparin, Origin of life, Macmillan, New York, 1938. 


\section{Directed Growth of Biomimetic}

[6] A.I. Oparin, K.L. Gladilin, Evolution of self-assembly of probionts, Biosystems 1980, 12, 133145.

[7] S.F. Banani, H.O. Lee, A.A. Hyman, M.K. Rosen, Biomolecular condensates: organizers of cellular biochemistry, Nature Reviews Molecular Cell Biology 2017, 18, 285-298.

Julicher, A.A. Hyman, Germline P Granules Are Liquid Droplets That Localize by Controlled Dissolution/Condensation, Science 2009, 324, 1729-1732.

[9] C.P. Brangwynne, T.J. Mitchison, A.A. Hyman, Active liquid-like behavior of nucleoli

determines their size and shape in Xenopus laevis oocytes, Proceedings of the National Academy of Sciences of the United States of America 2011, 108, 4334-4339.

[10] A.A. Hyman, C.A. Weber, F. Juelicher, Liquid-Liquid Phase Separation in Biology, in: R. Schekman, R. Lehmann (Eds.) Annual Review of Cell and Developmental Biology, Vol 302014, pp. 39-58.

[11] P.L. Li, S. Banjade, H.C. Cheng, S. Kim, B. Chen, L. Guo, M. Llaguno, J.V. Hollingsworth, D.S. King, S.F. Banani, P.S. Russo, Q.X. Jiang, B.T. Nixon, M.K. Rosen, Phase transitions in the assembly of multivalent signalling proteins, Nature 2012, 483, 336-U129.

[12] Y. Shin, C.P. Brangwynne, Liquid phase condensation in cell physiology and disease, Science 2017, 357.

Rosen, R.D. Vale, Phase separation of signaling molecules promotes $\mathrm{T}$ cell receptor signal transduction, Science 2016, 352, 595-599.

[14] S. Elbaum-Garfinkle, Y. Kim, K. Szczepaniak, C.C.H. Chen, C.R. Eckmann, S. Myong, C.P. Brangwynne, The disordered $\mathrm{P}$ granule protein LAF-1 drives phase separation into droplets with tunable viscosity and dynamics, Proceedings of the National Academy of Sciences of the United States of America 2015, 112, 7189-7194.

[15] S. Saha, C.A. Weber, M. Nousch, O. Adame-Arana, C. Hoege, M.Y. Hein, E. OsborneNishimura, J. Mahamid, M. Jahnel, L. Jawerth, A. Pozniakovski, C.R. Eckmann, F. Julicher, A.A. Hyman, Polar Positioning of Phase-Separated Liquid Compartments in Cells Regulated by an mRNA Competition Mechanism, Cell 2016, 166, 1572-+.

[16] K.A. Burke, A.M. Janke, C.L. Rhine, N.L. Fawzi, Residue-by-Residue View of In Vitro FUS Granules that Bind the C-Terminal Domain of RNA Polymerase II, Molecular Cell 2015, 60, 231241.

[17] T. Murakami, S. Qamar, J.Q. Lin, G.S.K. Schierle, E. Rees, A. Miyashita, A.R. Costa, R.B. Dodd, F.T.S. Chan, C.H. Michel, D. Kronenberg-Versteeg, Y. Li, S.P. Yang, Y. Wakutani, W. Meadows, R.R. Ferry, L. Dong, G.G. Tartaglia, G. Favrin, W.L. Lin, D.W. Dickson, M. Zhen, D. Ron, G. Schmitt-Ulms, P.E. Fraser, N.A. Shneider, C. Holt, M. Vendruscolo, C.F. Kaminski, P. St George-Hyslop, ALS/FTD Mutation-Induced Phase Transition of FUS Liquid Droplets and Reversible Hydrogels into Irreversible Hydrogels Impairs RNP Granule Function, Neuron 2015, 88, 678-690. Saha, T.M. Franzmann, A. Pozniakovski, I. Poser, N. Maghelli, L.A. Royer, M. Weigert, E.W. Myers, S. Grill, D. Drechsel, A.A. Hyman, S. Alberti, A Liquid-to-Solid Phase Transition of the ALS Protein FUS Accelerated by Disease Mutation, Cell 2015, 162, 1066-1077.

[19] J.B. Woodruff, B.F. Gomes, P.O. Widlund, J. Mahamid, A. Honigmann, A.A. Hyman, The 
[20] M. Feric, N. Vaidya, T.S. Harmon, D.M. Mitrea, L. Zhu, T.M. Richardson, R.W. Kriwacki, R.V. Pappu, C.P. Brangwynne, Coexisting Liquid Phases Underlie Nucleolar Subcompartments, Cell 2016, 165, 1686-1697. [21] A. Hubstenberger, S.L. Noble, C. Cameron, T.C. Evans, Translation Repressors, an RNA Helicase, and Developmental Cues Control RNP Phase Transitions during Early Development, Developmental Cell 2013, 27, 161-173. Richter, W. Baumeister, S.W. Grill, R.V. Pappu, A.A. Hyman, S. Alberti, Phase separation of a yeast prion protein promotes cellular fitness, Science 2018, 359, 47-+.

[23] J. Wang, J.M. Choi, A.S. Holehouse, H.O. Lee, X.J. Zhang, M. Jahnel, S. Maharana, R. Lemaitre, A. Pozniakovsky, D. Drechsel, I. Poser, R.V. Pappu, S. Alberti, A.A. Hyman, A Molecular Grammar Governing the Driving Forces for Phase Separation of Prion-like RNA Binding Proteins, Cell 2018, 174, 688-+.

[24] S. Boeynaems, S. Alberti, N.L. Fawzi, T. Mittag, M. Polymenidou, F. Rousseau, J. Schymkowitz, J. Shorter, B. Wolozin, L. Van den Bosch, P. Tompa, M. Fuxreiter, Protein Phase Separation: A New Phase in Cell Biology, Trends in Cell Biology 2018, 28, 420-435.

[25] T.J. Nott, E. Petsalaki, P. Farber, D. Jervis, E. Fussner, A. Plochowietz, T.D. Craggs, D.P. Bazett-Jones, T. Pawson, J.D. Forman-Kay, A.J. Baldwin, Phase Transition of a Disordered Nuage Protein Generates Environmentally Responsive Membraneless Organelles, Molecular Cell 2015, 57, 936-947.

[26] T.S. Harmon, A.S. Holehouse, M.K. Rosen, R.V. Pappu, Intrinsically disordered linkers determine the interplay between phase separation and gelation in multivalent proteins, Elife 2017, 6 . [27] A. Molliex, J. Temirov, J. Lee, M. Coughlin, A.P. Kanagaraj, H.J. Kim, T. Mittag, J.P. Taylor, Phase Separation by Low Complexity Domains Promotes Stress Granule Assembly and Drives Pathological Fibrillization, Cell 2015, 163, 123-133.

[28] D. Zwicker, A.A. Hyman, F. Julicher, Suppression of Ostwald ripening in active emulsions, Physical Review E 2015, 92.

[29] D. Zwicker, R. Seyboldt, C.A. Weber, A.A. Hyman, F. Julicher, Growth and division of active droplets provides a model for protocells, Nature Physics 2017, 13, 408-413.

[30] T. Franzmann, S. Alberti, Prion-like low-complexity sequences: Key regulators of protein solubility and phase behavior, J Biol Chem 2018.

[31] K.M. Ruff, S. Roberts, A. Chilkoti, R.V. Pappu, Advances in Understanding Stimulus Responsive Phase Behavior of Intrinsically Disordered Protein Polymers, J Mol Biol 2018.

[32] R. Chollakup, W. Smitthipong, C.D. Eisenbach, M. Tirrell, Phase Behavior and Coacervation of Aqueous Poly(acrylic acid)-Poly(allylamine) Solutions, Macromolecules 2010, 43, 2518-2528.

[33] T.Y.D. Tang, C.R.C. Hak, A.J. Thompson, M.K. Kuimova, D.S. Williams, A.W. Perriman, S. Mann, Fatty acid membrane assembly on coacervate microdroplets as a step towards a hybrid protocell model, Nature Chemistry 2014, 6, 527-533.

[34] K.A. Black, D. Priftis, S.L. Perry, J. Yip, W.Y. Byun, M. Tirrell, Protein Encapsulation via Polypeptide Complex Coacervation, Acs Macro Letters 2014, 3, 1088-1091.

[35] D. Priftis, K. Megley, N. Laugel, M. Tirrell, Complex coacervation of poly(ethyleneimine)/polypeptide aqueous solutions: Thermodynamic and rheological characterization, Journal of Colloid and Interface Science 2013, 398, 39-50.

[36] D. Priftis, M. Tirrell, Phase behaviour and complex coacervation of aqueous polypeptide solutions, Soft Matter 2012, 8, 9396-9405.

[37] W.M. Aumiller, C.D. Keating, Phosphorylation-mediated RNA/peptide complex coacervation as a model for intracellular liquid organelles, Nature Chemistry 2016, 8, 129-137. 
[38] J.R. Vieregg, M. Lueckheide, A.B. Marciel, L. Leon, A.J. Bologna, J.R. Rivera, M.V. Tirrell, Oligonucleotide-Peptide Complexes: Phase Control by Hybridization, Journal of the American Chemical Society 2018, 140, 1632-1638.

[39] S. Koga, D.S. Williams, A.W. Perriman, S. Mann, Peptide-nucleotide microdroplets as a step towards a membrane-free protocell model, Nature Chemistry 2011, 3, 720-724.

[40] R. Dimova, R. Lipowsky, Lipid membranes in contact with aqueous phases of polymer solutions, Soft Matter 2012, 8, 6409-6415.

[41] C.D. Keating, Aqueous Phase Separation as a Possible Route to Compartmentalization of Biological Molecules, Accounts of Chemical Research 2012, 45, 2114-2124.

R.V. Pappu, M.K. Rosen, Sequence Determinants of Intracellular Phase Separation by Complex Coacervation of a Disordered Protein, Molecular Cell 2016, 63, 72-85.

[43] V.N. Uversky, I.M. Kuznetsova, K.K. Turoverov, B. Zaslavsky, Intrinsically disordered proteins as crucial constituents of cellular aqueous two phase systems and coacervates, Febs Letters 2015, $589,15-22$.

[44] E. Spruijt, J. Sprakel, M.A.C. Stuart, J. van der Gucht, Interfacial tension between a complex coacervate phase and its coexisting aqueous phase, Soft Matter 2010, 6, 172-178.

[45] Y.G. Liu, R. Lipowsky, R. Dimova, Concentration Dependence of the Interfacial Tension for Aqueous Two-Phase Polymer Solutions of Dextran and Polyethylene Glycol, Langmuir 2012, 28, 3831-3839.

[46] D.S. Williams, S. Koga, C.R.C. Hak, A. Majrekar, A.J. Patil, A.W. Perriman, S. Mann, Polymer/nucleotide droplets as bio-inspired functional micro-compartments, Soft Matter 2012, 8, 6004-6014.

[47] E.A. Frankel, P.C. Bevilacqua, C.D. Keating, Polyamine/Nucleotide Coacervates Provide Strong Compartmentalization of Mg2+, Nucleotides, and RNA, Langmuir 2016, 32, 2041-2049.

[48] T.Y.D. Tang, M. Antognozzi, J.A. Vicary, A.W. Perriman, S. Mann, Small-molecule uptake in membrane-free peptide/nucleotide protocells, Soft Matter 2013, 9, 7647-7656.

[49] M.S. Long, C.D. Jones, M.R. Helfrich, L.K. Mangeney-Slavin, C.D. Keating, Dynamic microcompartmentation in synthetic cells, Proc. Natl. Acad. Sci. U. S. A. 2005, 102, 5920-5925.

[50] E. Sokolova, E. Spruijt, M.M.K. Hansen, E. Dubuc, J. Groen, V. Chokkalingam, A. Piruska, H.A. Heus, W.T.S. Huck, Enhanced transcription rates in membrane-free protocells formed by coacervation of cell lysate, Proceedings of the National Academy of Sciences of the United States of America 2013, 110, 11692-11697.

[51] T.Y.D. Tang, D. van Swaay, A. deMello, J.L.R. Anderson, S. Mann, In vitro gene expression within membrane-free coacervate protocells, Chemical Communications 2015, 51, 11429-11432.

[52] K.K. Nakashima, J.F. Baaij, E. Spruijt, Reversible generation of coacervate droplets in an enzymatic network, Soft Matter 2018, 14, 361-367.

[53] P. Stano, P.L. Luisi, Achievements and open questions in the self-reproduction of vesicles and synthetic minimal cells, Chem Commun (Camb) 2010, 46, 3639-3653.

[54] M.M. Hanczyc, S.M. Fujikawa, J.W. Szostak, Experimental models of primitive cellular compartments: Encapsulation, growth, and division, Science 2003, 302, 618-622.

[55] D. Deamer, J.P. Dworkin, S.A. Sandford, M.P. Bernstein, L.J. Allamandola, The first cell membranes, Astrobiology 2002, 2, 371-381.

[56] N.R.C. Steering Group for the Workshop on Size Limits of Very Small Microorganisms, Size Limits of Very Small Microorganisms: Proceedings of a Workshop, National Academies Press (US)

Copyright 1999 by the National Academy of Sciences. All rights reserved., Washington (DC), 1999. 
[57] T.P. de Souza, P. Stano, P.L. Luisi, The Minimal Size of Liposome-Based Model Cells Brings about a Remarkably Enhanced Entrapment and Protein Synthesis, Chembiochem 2009, 10, 10561063.

[58] R. Dimova, S. Aranda, N. Bezlyepkina, V. Nikolov, K.A. Riske, R. Lipowsky, A practical guide to giant vesicles. Probing the membrane nanoregime via optical microscopy, J. Phys.: Condens. Matter 2006, 18, S1151-S1176.

[59] R. Dimova, Giant Vesicles: A Biomimetic Tool for Membrane Characterization, in: A. Iglič (Ed.) Advances in Planar Lipid Bilayers and Liposomes, Academic Press2012, pp. 1-50.

[60] P. Walde, K. Cosentino, H. Engel, P. Stano, Giant Vesicles: Preparations and Applications, ChemBioChem 2010, 11, 848-865.

[61] S.F. Fenz, K. Sengupta, Giant vesicles as cell models, Integr Biol-Uk 2012, 4, 982-995.

[62] K. Morigaki, P. Walde, Fatty acid vesicles, Current Opinion in Colloid \& Interface Science 2007, 12, 75-80.

[63] I.A. Chen, R.W. Roberts, J.W. Szostak, The Emergence of Competition Between Model Protocells, Science 2004, 305, 1474.

[64] P. Walde, R. Wick, M. Fresta, A. Mangone, P.L. Luisi, Autopoietic Self-Reproduction of Fatty Acid Vesicles, J. Am. Chem. Soc. 1994, 116, 11649-11654.

[65] E. Blochliger, M. Blocher, P. Walde, P.L. Luisi, Matrix effect in the size distribution of fatty acid vesicles, Journal of Physical Chemistry B 1998, 102, 10383-10390.

[66] I.A. Chen, J.W. Szostak, A kinetic study of the growth of fatty acid vesicles, Biophys J 2004, 87, 988-998.

[67] A.J. Markvoort, N. Pfleger, R. Staffhorst, P.A. Hilbers, R.A. van Santen, J.A. Killian, B. de Kruijff, Self-reproduction of fatty acid vesicles: a combined experimental and simulation study, Biophys J 2010, 99, 1520-1528.

[68] C. Hentrich, J.W. Szostak, Controlled growth of filamentous fatty acid vesicles under flow, Langmuir 2014, 30, 14916-14925.

[69] A.E. Engelhart, K.P. Adamala, J.W. Szostak, A simple physical mechanism enables homeostasis in primitive cells, Nature Chemistry 2016, 8, 448-453.

[70] T.F. Zhu, J.W. Szostak, Coupled Growth and Division of Model Protocell Membranes, Journal of the American Chemical Society 2009, 131, 5705-5713.

[71] A. Shioi, T.A. Hatton, Model for formation and growth of vesicles in mixed anionic/cationic (SOS/CTAB) surfactant systems, Langmuir 2002, 18, 7341-7348.

[72] N. Berclaz, M. Muller, P. Walde, P.L. Luisi, Growth and transformation of vesicles studied by ferritin labeling and cryotransmission electron microscopy, Journal of Physical Chemistry B 2001, 105, 1056-1064.

[73] T.P. de Souza, M. Holzer, P. Stano, F. Steiniger, S. May, R. Schubert, A. Fahr, P.L. Luisi, New 47591 Insights into the Growth and Transformation of Vesicles: A Free-Flow Electrophoresis Study, Journal of Physical Chemistry B 2015, 119, 12212-12223. acids with phosphatidylcholine vesicles (liposomes), Colloids Surf B Biointerfaces 2006, 48, 24-34. [75] P. Peterlin, V. Arrigler, K. Kogej, S. Svetina, P. Walde, Growth and shape transformations of giant phospholipid vesicles upon interaction with an aqueous oleic acid suspension, Chemistry and Physics of Lipids 2009, 159, 67-76.

[76] J. Dervaux, V. Noireaux, A.J. Libchaber, Growth and instability of a phospholipid vesicle in a bath of fatty acids, The European Physical Journal Plus 2017, 132. 
[78] V.N. Georgiev, A. Grafmüller, D. Bléger, S. Hecht, S. Kunstmann, S. Barbirz, R. Lipowsky, R. Dimova, Area Increase and Budding in Giant Vesicles Triggered by Light: Behind the Scene, Advanced Science 2018, 5, 1800432.

7606

[79] R. Dasgupta, M.S. Miettinen, N. Fricke, R. Lipowsky, R. Dimova, The glycolipid GM1 reshapes asymmetric biomembranes and giant vesicles by curvature generation, Proc. Natl. Acad. Sci. U. S. A. 2018, 115, 5756-5761.

[80] R. Lipowsky, Spontaneous tubulation of membranes and vesicles reveals membrane tension generated by spontaneous curvature, Faraday Discuss. 2013, 161, 305-331.

[81] P. Bassereau, R. Jin, T. Baumgart, M. Deserno, R. Dimova, V.A. Frolov, P.V. Bashkirov, H. Grubmüller, R. Jahn, H.J. Risselada, L. Johannes, M.M. Kozlov, R. Lipowsky, T.J. Pucadyil, W.F. Zeno, J.C. Stachowiak, D. Stamou, A. Breuer, L. Lauritsen, C. Simon, C. Sykes, G.A. Voth, T.R. Weikl, The 2018 biomembrane curvature and remodeling roadmap, J. Phys. D: Appl. Phys. 2018, 51, 343001.

[82] Y. Inaoka, M. Yamazaki, Vesicle fission of giant unilamellar vesicles of liquid-ordered-phase membranes induced by amphiphiles with a single long hydrocarbon chain, Langmuir 2007, 23, 720728.

[83] B. Bozic, S. Svetina, A relationship between membrane properties forms the basis of a selectivity mechanism for vesicle self-reproduction, European Biophysics Journal with Biophysics Letters 2004, 33, 565-571.

[84] D. Lichtenberg, H. Ahyayauch, F.M. Goni, The Mechanism of Detergent Solubilization of Lipid Bilayers, Biophysical Journal 2013, 105, 289-299.

[85] B. Mattei, R.B. Lira, K.R. Perez, K.A. Riske, Membrane permeabilization induced by Triton X100: The role of membrane phase state and edge tension, Chemistry and Physics of Lipids 2017, 202, 28-37.

[86] F. Caschera, P. Stano, P.L. Luisi, Reactivity and fusion between cationic vesicles and fatty acid anionic vesicles, Journal of Colloid and Interface Science 2010, 345, 561-565.

[87] C.K. Haluska, K.A. Riske, V. Marchi-Artzner, J.M. Lehn, R. Lipowsky, R. Dimova, Time scales of membrane fusion revealed by direct imaging of vesicle fusion with high temporal resolution, Proc Natl Acad Sci U S A 2006, 103, 15841-15846.

[88] R.B. Lira, T. Robinson, R. Dimova, K.A. Riske, Charge-dependent membrane fusion in a pure lipid system: a single giant vesicle study, Biophys $J$ 2018, (in press).

[89] V.P. Zhdanov, B. Kasemo, Lipid-diffusion-limited kinetics of vesicle growth, Langmuir 2000, $16,7352-7354$.

[90] B. Bozic, S. Svetina, Vesicle self-reproduction: The involvement of membrane hydraulic and solute permeabilities, European Physical Journal E 2007, 24, 79-90.

[91] D. Fanelli, A.J. McKane, Thermodynamics of vesicle growth and instability, Physical Review E 2008, 78 .

[92] F. Mavelli, E. Altamura, L. Cassidei, P. Stano, Recent Theoretical Approaches to Minimal Artificial Cells, Entropy 2014, 16, 2488-2511.

[93] P.K. Schmidli, P. Schurtenberger, P.L. Luisi, Liposome-mediated enzymatic-synthesis of phosphatidylcholine as an approach to self-replicating liposomes, Journal of the American Chemical Society 1991, 113, 8127-8130.

[94] Y. Kuruma, P. Stano, T. Ueda, P.L. Luisi, A synthetic biology approach to the construction of membrane proteins in semi-synthetic minimal cells, Biochim Biophys Acta 2009, 1788, 567-574.

[95] A. Scott, M.J. Noga, P. de Graaf, I. Westerlaken, E. Yildirim, C. Danelon, Cell-Free Phospholipid Biosynthesis by Gene-Encoded Enzymes Reconstituted in Liposomes, Plos One 2016, 11. 
[96] G. Murtas, Internal lipid synthesis and vesicle growth as a step toward self-reproduction of the minimal cell, Syst Synth Biol 2010, 4, 85-93.

[97] M. Exterkate, A. Caforio, M.C.A. Stuart, A.J.M. Driessen, Growing Membranes In Vitro by Continuous Phospholipid Biosynthesis from Free Fatty Acids, Acs Synthetic Biology 2018, 7, 153165.

[98] K. Kurihara, M. Tamura, K. Shohda, T. Toyota, K. Suzuki, T. Sugawara, Self-reproduction of supramolecular giant vesicles combined with the amplification of encapsulated DNA, Nat Chem 2011, 3, 775-781. reproducing catalyst drives repeated phospholipid synthesis and membrane growth, Proceedings of the National Academy of Sciences of the United States of America 2015, 112, 8187-8192.

[100] T. Tanaka, M. Yamazaki, Membrane fusion of giant unilamellar vesicles of neutral phospholipid membranes induced by La3+, Langmuir 2004, 20, 5160-5164.

[101] D.P. Pantazatos, R.C. MacDonald, Directly observed membrane fusion between oppositely charged phospholipid bilayers, Journal of Membrane Biology 1999, 170, 27-38.

[102] T. Sunami, F. Caschera, Y. Morita, T. Toyota, K. Nishimura, T. Matsuura, H. Suzuki, M.M. Hanczyc, T. Yomo, Detection of Association and Fusion of Giant Vesicles Using a FluorescenceActivated Cell Sorter, Langmuir 2010, 26, 15098-15103.

[103] B.R. Lentz, PEG as a tool to gain insight into membrane fusion, Eur Biophys J 2007, 36, 315 326.

[104] D. Tareste, J. Shen, T.J. Melia, J.E. Rothman, SNAREpin/Munc18 promotes adhesion and fusion of large vesicles to giant membranes, Proceedings of the National Academy of Sciences of the United States of America 2008, 105, 2380-2385.

[105] M. Kyoung, Y. Zhang, J. Diao, S. Chu, A.T. Brunger, Studying calcium-triggered vesicle fusion in a single vesicle-vesicle content and lipid-mixing system, Nat Protoc 2013, 8, 1-16.

[106] Y.H.M. Chan, B. van Lengerich, S.G. Boxer, Lipid-anchored DNA mediates vesicle fusion as observed by lipid and content mixing, Biointerphases 2008, 3, FA17-FA21.

[107] A. Rorvig-Lund, A. Bahadori, S. Semsey, P.M. Bendix, L.B. Oddershede, Vesicle Fusion Triggered by Optically Heated Gold Nanoparticles, Nano Letters 2015, 15, 4183-4188. Membranes, Langmuir 2017, 33, 2671-2676. budding of giant phospholipid vesicles containing macromolecules, Proc Natl Acad Sci U S A 2012, 109, 5942-5947.

[110] K.A. Riske, N. Bezlyepkina, R. Lipowsky, R. Dimova, Electrofusion of model lipid membranes viewed with high temporal resolution, Biophys. Rev. Lett. 2006, 1, 387-400.

[111] J.C. Shillcock, R. Lipowsky, Tension-induced fusion of bilayer membranes and vesicles, Nat. Mater. 2005, 4, 225-228.

[112] D.K. Struck, D. Hoekstra, R.E. Pagano, Use of resonance energy-transfer to monitor membrane fusion, Biochemistry 1981, 20, 4093-4099.

[113] J. Wilschut, N. Duzgunes, R. Fraley, D. Papahadjopoulos, Studies on the mechanism of membrane-fusion - kinetics of calcium-ion induced fusion of phosphatidylserine vesicles followed by a new assay for mixing of aqueous vesicle contents, Biochemistry 1980, 19, 6011-6021.

[114] G.H. Lei, R.C. MacDonald, Lipid bilayer vesicle fusion: Intermediates captured by high-speed microfluorescence spectroscopy, Biophysical Journal 2003, 85, 1585-1599.

[115] A. Witkowska, R. Jahn, Rapid SNARE-Mediated Fusion of Liposomes and Chromaffin Granules with Giant Unilamellar Vesicles, Biophysical Journal 2017, 113, 1251-1259. 
[116] T.-T. Kliesch, J. Dietz, L. Turco, P. Halder, E. Polo, M. Tarantola, R. Jahn, A. Janshoff,

Membrane tension increases fusion efficiency of model membranes in the presence of SNAREs, Sci Rep 2017, 7, 12070.

[117] S. Trier, J.R. Henriksen, T.L. Andresen, Membrane fusion of pH-sensitive liposomes - a quantitative study using giant unilamellar vesicles, Soft Matter 2011, 7, 9027-9034. [118] J. Solon, J. Pecreaux, P. Girard, M.C. Faure, J. Prost, P. Bassereau, Negative tension induced by lipid uptake, Phys. Rev. Lett. 2006, 97, 098103.

[119] D.T. Chiu, C.F. Wilson, F. Ryttsen, A. Stromberg, C. Farre, A. Karlsson, S. Nordholm, A. Gaggar, B.P. Modi, A. Moscho, R.A. Garza-Lopez, O. Orwar, R.N. Zare, Chemical transformations in individual ultrasmall biomimetic containers, Science 1999, 283, 1892-1895.

[120] T. Robinson, P.E. Verboket, K. Eyer, P.S. Dittrich, Controllable electrofusion of lipid vesicles: initiation and analysis of reactions within biomimetic containers, Lab on a Chip 2014, 14, 28522859.

[121] N. Bezlyepkina, R.S. Gracia, P. Shchelokovskyy, R. Lipowsky, R. Dimova, Phase Diagram and Tie-Line Determination for the Ternary Mixture DOPC/eSM/Cholesterol, Biophysical Journal 2013, 104, 1456-1464.

[122] S. Kulin, R. Kishore, K. Helmerson, L. Locascio, Optical manipulation and fusion of liposomes as microreactors, Langmuir 2003, 19, 8206-8210.

[123] P. Yang, R. Lipowsky, R. Dimova, Nanoparticle formation in giant vesicles: synthesis in biomimetic compartments, Small 2009, 5, 2033-2037.

[124] J. Nikolaus, M. Stöckl, D. Langosch, R. Volkmer, A. Herrmann, Direct Visualization of Large and Protein-Free Hemifusion Diaphragms, Biophys. J. 2010, 98, 1192-1199.

[125] J. Heuvingh, F. Pincet, S. Cribier, Hemifusion and fusion of giant vesicles induced by reduction of inter-membrane distance, The European Physical Journal E 2004, 14, 269-276.

[126] A.C. Saito, T. Ogura, K. Fujiwara, S. Murata, S.M. Nomura, Introducing Micrometer-Sized Artificial Objects into Live Cells: A Method for Cell-Giant Unilamellar Vesicle Electrofusion, Plos One 2014, 9.

[127] A. Strömberg, F. Ryttsén, D.T. Chiu, M. Davidson, P.S. Eriksson, C.F. Wilson, O. Orwar, R.N. Zare, Manipulating the genetic identity and biochemical surface properties of individual cells with electric-field-induced fusion, Proceedings of the National Academy of Sciences 2000, 97, 7.

[128] B.M. Discher, Y.Y. Won, D.S. Ege, J.C.M. Lee, F.S. Bates, D.E. Discher, D.A. Hammer, Polymersomes: Tough vesicles made from diblock copolymers, Science 1999, 284, 1143-1146.

[129] J.C.M. Lee, M. Santore, F.S. Bates, D.E. Discher, From membranes to melts, rouse to reptation: Diffusion in polymersome versus lipid bilayers, Macromolecules 2002, 35, 323-326.

[130] R. Dimova, U. Seifert, B. Pouligny, S. Forster, H.G. Dobereiner, Hyperviscous diblock copolymer vesicles, Eur. Phys. J. E 2002, 7, 241-250.

[131] Y.F. Zhou, D.Y. Yan, Real-time membrane fusion of giant polymer vesicles, Journal of the American Chemical Society 2005, 127, 10468-10469.

[132] W. Su, Y.H. Luo, Q. Yan, S. Wu, K. Han, Q.J. Zhang, Y.Q. Gu, Y.M. Li, Photoinduced fusion of micro-vesicles self-assembled from azobenzene-containing amphiphilic diblock copolymers, Macromolecular Rapid Communications 2007, 28, 1251-1256.

[133] T.P. Smart, C. Fernyhough, A.J. Ryan, G. Battaglia, Controlling Fusion and Aggregation in Polymersome Dispersions, Macromolecular Rapid Communications 2008, 29, 1855-1860.

[134] J.W. Szostak, D.P. Bartel, P.L. Luisi, Synthesizing life, Nature 2001, 409, 387-390.

[135] P.L. Luisi, T.P. de Souza, P. Stano, Vesicle Behavior: In Search of Explanations, Journal of Physical Chemistry B 2008, 112, 14655-14664.

[136] J.E. Rothman, F.T. Wieland, Protein sorting by transport vesicles, Science 1996, 272, 227-234. 


\section{Directed Growth of Biomimetic Microcompartments}

${ }_{3}^{3} 44$ [137] F. Caschera, T. Sunami, T. Matsuura, H. Suzuki, M.M. Hanczyc, T. Yomo, Programmed 545 Vesicle Fusion Triggers Gene Expression, Langmuir 2011, 27, 13082-13090.

${ }_{6}^{746}$ [138] K.P. Browne, D.A. Walker, K.J.M. Bishop, B.A. Grzybowski, Self-Division of Macroscopic 7747 Droplets: Partitioning of Nanosized Cargo into Nanoscale Micelles, Angewandte Chemie8748 International Edition 2010, 49, 6756-6759.

949 [139] F. Caschera, S. Rasmussen, M.M. Hanczyc, An Oil Droplet Division-Fusion Cycle, ${ }_{11}^{10} 750 \quad$ Chempluschem 2013, 78, 52-54. 